\title{
ENFOQUE ANTITÉTICO DEL OCIO
}

\section{ANTITHETICAL APPROACH TO LEISURE}

(iD) Luis Alberto Bretoneche Gutiérrez ${ }^{*}$

labguni@gmail.com

${ }^{1}$ Universidad Nacional Mayor de San Marcos, Lima, Perú

*Correspondencia: Luis Alberto Bretoneche Gutiérrez. Email: labguni@gmail.com

Recibido: 15.04.2020 | Aprobado: 18.05.2020

\section{RESUMEN}

A través de este texto se podrán apreciar opiniones antitéticas del término ocio o sus equivalentes que tienen implicancias en el conocimiento, la vida cotidiana, la ciencia y la filosofía. Los dos sentidos que ha tenido el término ocio a través del tiempo son el negativo y el positivo. Se presenta resumidamente, por un lado, el sentido negativo que le dan las personas comunes, Hesíodo, Séneca, Michel de Montaigne, Francis Bacon, Immanuel Kant y Frederick W. Taylor, mientras que, por otra parte, se podrá estimar el sentido positivo del concepto en Platón, Aristóteles y Cicerón.

Palabras clave: Haraganeo, holgazanería, ocio.

\section{ABSTRACT}

Through this text, you can see antithetical opinions of the term leisure or its equivalents that have implications in knowledge, daily life, science and philosophy. The two senses that the term leisure has had over time are the negative and the positive. It is summarized, on the one hand, the negative sense given by ordinary people, Hesiod, Seneca, Michel de Montaigne, Francis Bacon, Immanuel Kant and Frederick W. Taylor, while, on the other hand, you can estimate the positive sense of the concept in Plato, Aristotle and Cicero.

Keywords: Idle, laziness, leisure. 


\section{INTRODUCCIÓN}

En este artículo se presentan los significados positivos y negativos que ha tenido el término ocio a través del tiempo y en palabras de diferentes autores, con la finalidad de mostrar la relación antitética del concepto. Este examen y reflexión se realizará sabiendo que existen, por lo menos, dos sentidos de este término que aparecen tanto en Immanuel Kant como en Frederick Engels. La primera tarea que se presenta es aclarar el uso del término antitético. Por ejemplo, Kant (1984) afirmó que: "Si tética es todo conjunto de doctrinas dogmáticas, entiendo por antitética, no las afirmaciones dogmáticas de lo opuesto, sino el conflicto de conocimientos al parecer dogmáticos... sin que uno tenga más derecho que otro a ser aplaudido" (p. 321), mientras que Engels (1971) sostuvo lo siguiente: "Asimismo, nos encontramos, observando las cosas detenidamente, con que los dos polos de una antítesis, el positivo y el negativo, son tan inseparables como antitéticos el uno del otro y que, pese a todo su antagonismo, se penetran recíprocamente..." (p. 48). Interpretando las definiciones tan divergentes de estos dos filósofos, se puede observar que para el primer pensador el término antitético expresa, además de las afirmaciones distintas de los dogmáticos, un conflicto de conocimientos de igual valor que el expresado por los dogmáticos y que, por ende, tiene que ser considerado como una crítica de la razón misma o, dicho de otro modo, un uso libre de la razón: “... la antitética no se ocupa de afirmaciones unilaterales, sino se considera conocimientos universales de la razón en su conflicto y en las causas de ese conflicto" (Kant, 1984, p. 321); mientras que, para el segundo pensador, la palabra antitético expresa una relación ontológica distinta a la relación gnoseológica expresada por Kant, esta relación de unidad de los opuestos se ve marcada por los valores positivos y negativos que se encuentra intrínsecamente en los objetos, cosas o entes, y, además, en los hechos o sucesos de naturaleza material o espiritual; es decir, lo antitético expresa una relación de aceptación y rechazo de la realidad tanto simple como compleja, en la cual las diferencias se penetran o conviven entre sí. A pesar de estas diferencias, las dos concepciones de antitético no se oponen en el uso que se hace del término en este texto, por ende, desde esta perspectiva, se va a iniciar un estudio y reflexión sobre el sentido negativo del término ocio para luego pasar al enfoque positivo.

Según el Diccionario latino-español español-latino (1958), el sustantivo neutro latino otĩum (ocio, descanso, paz, tranquilidad, sosiego y consagrado al estudio) deriva del adjetivo otiōsus que tiene también muchas traducciones: "ocioso, desocupado; libre de los asuntos públicos; neutral, indiferente; tranquilo, apacible; pausado; inútil y superfluo” (p. 286). Estos significados castellanos de la lengua latina envuelven, como se pueden apreciar, la relación antitética del término, ya que se pueden percibir en los significados tanto la valoración positiva como la negativa, es decir, los dos sentidos, aunque hay un mayor número de valoraciones negativas. Esta relación es la que, tal vez, haya influido o generado que, en la actualidad, el significado peyorativo del término ocio sea el más conocido, notorio o empleado, en otras palabras, este sería el sentido común del término. Además, se puede observar que esta opinión vulgar tenga una mayor carga en el imaginario colectivo de la gente, motivo por el cual viene siendo utilizado por el común denominador de las personas desde tiempos antiguos. Por ende, es frecuente oír a las personas adultas amonestar como ociosos a los jóvenes y niños por su poca o nula dedicación al trabajo o 
estudio. Los calificativos que surgen en esta situación son los de vago, perezoso u ocioso. A partir de esta perspectiva es que en este trabajo se comienza explorando, en primer lugar, el enfoque peyorativo o negativo que ha tenido el término en cuestión.

\section{CONCEPCIÓN NEGATIVA DEL TÉRMINO OCIO}

El concepto de ocio como sinónimo de holgazanería, flojera o tedio ha sido siempre motivo de recriminación incluso en la antigüedad griega, un ejemplo de esta afirmación se observó en Hesíodo (2000): "Por los trabajos se hacen los hombres ricos en ganado y opulentos, y si trabajas te apreciarán mucho más los Inmortales [y los mortales; pues aborrecen en gran manera a los holgazanes]" (p. 81). Este vate griego continuó con sus observaciones acerca del valor positivo del trabajo sobre la inoperancia del ocio: "El trabajo no es ninguna deshonra; la inactividad es una deshonra. Si trabajas pronto te tendrá envidia el indolente al hacerte rico. La valía y la estimación van unidas al dinero" (Hesíodo, p. 81).

Con respecto a lo mencionado, se puede percibir que en el lenguaje de Hesíodo se presenta el antagonismo de dos conceptos: el ocioso u holgazán frente al trabajador u hombre exitoso en la vida. La actitud del primero sólo le trae deshonra y repudio; mientras que, para el segundo, su modo de actuar trae honor y riqueza, es decir, es una persona virtuosa. En consecuencia, se puede interpretar que, para este autor, el trabajo no sólo traería dinero, riquezas o reconocimiento, sino también, areté o virtud; mientras que, el ocio sería todo lo opuesto.

Se puede incluir en esta concepción negativa del término ocio a la tradición filosófica de la lengua latina, donde se puede prestar atención especial a la posición de Séneca (2005) cuando afirmó que:

El tiempo que tenemos no es corto; pero, perdiendo mucho de él, hacemos lo que sea, y la vida es suficientemente larga para ejecutar en ella cosas grandes, si la empleáramos bien. Pero al que se le pasa en ocio y en placeres, y no la ocupa en loables ejercicios, cuando le llega el último trance, conocemos que se le fue, sin que él haya entendido que caminaba. (p. 19)

Aunque se puede poner atención a las palabras de Séneca, respecto a la relación que existe entre tiempo y ocio, donde este último término en cuestión sólo sería hacer un mal uso del tiempo o desperdiciarlo; es decir, se percibe que el autor asocia esta actitud a llevar una vida dada a los placeres que se pueden calificar como mundanos. Luego va a señalar que la consecuencia natural de esta pérdida de tiempo es la torpeza:

¿Para qué nos quejamos de la naturaleza, pues ella se hubo con nosotros benignamente? Larga es la vida, si la sabemos aprovechar. A uno detiene la insaciable avaricia; a otro, la cuidadosa diligencia de inútiles trabajos; uno se entrega al vino, otro con la ociosidad la entorpece... (Séneca 2005, p. 20)

Finalmente, este filósofo romano culmina con una reflexión y asociación negativa de ideas donde se distancia la relación entre ocio y ocioso llevando in extremo el término ocioso hacia una posición negativa, asociándolo a nociones como enfermedad, andar medio vivo o, peor aún, andar muerto por el mundo: 
Éste, pues, no se debe llamar ocioso; otro nombre se le debe poner: enfermo está, o, por mejor decir, muerto. Ocioso es el que conoce su oficio; pero el que para entender sus acciones corporales necesita de quien se las advierta éste solamente es medio vivo. (Séneca 2005, p. 41)

Luego de los comentarios breves y los resúmenes dados a las citas presentadas, se puede sintetizar que, para Séneca, el ocioso tiene valoraciones que colindan con los aspectos negativos de la existencia humana, las cuales se pueden caracterizar o relacionar como perdedor del tiempo, de temperamento o naturaleza concupiscible, de andar torpe, enfermo y hasta de muerto por el mundo, entre otros. Todos estos calificativos llevan a la reflexión que no es bueno ser ocioso, es decir, tener la tendencia a practicar el ocio: Por otro lado, se deduce que lo bueno es todo lo contrario: ser laborioso, ejercitarse, es decir, no ocioso. Aunque es necesario resaltar que en Séneca, calificado como partidario de la concepción negativa de ocio u ocioso, se puede percibir un empleo antitético del término cuando expresa la distinción entre ocioso y hombre enfermo o muerto, claro que esta aclaración es interpretada como metafórica en este artículo.

De la misma opinión que Hesíodo y Séneca es la posición de Michel de Montaigne (1898) cuando en su ensayo De la ociosidad afirma que el ocio o no hacer nada nos lleva a una conducta desordenada: “... así acontece con los espíritus; si no se los ocupa en labor determinada que los sujete y contraiga se lanzan desordenadamente en el vago campo de las fantasías" (p. 21). Para este filósofo francés, la ociosidad implica, además de la conducta señalada, no tener un proyecto de vida y andar por el mundo desubicado: "El alma se pierde cuando no tiene un fin establecido, pues como suele decirse, estar en todas partes no es encontrarse en ninguna" (Montaigne, p. 21). La posición de este pensador francés es totalmente crítica ante las nociones de ocio y ociosidad, la cual lleva por sí sola a conducirse con actitudes negativas y por senderos obscuros:

Cuando el caballo escapa solo, toma cien veces más carrera que cuando el jinete lo conduce; mi espíritu ocioso engendra tantas quimeras, tantos monstruos fantásticos, sin darse tregua ni reposo, sin orden ni concierto, que para poder contemplar á [sic] mi gusto la ineptitud y singularidad de los mismos, he comenzado á [sic] ponerlos por escrito, esperando con el tiempo que se avergüence al contemplar imaginaciones tales. (Montaigne, 1898, pp. 21 y 22)

En pocas palabras, para Michael de Montaigne, el ocioso tiene un espíritu o alma fantasiosa, vaga, desubicada e inepta, entre otras características, y, en consecuencia, no es recomendable practicar esta conducta. Se puede observar, también, que esta crítica al ocio se da en el plano del empleo intelectual del tiempo.

Continuando con lo ilustrado, tanto para los que manejen una posición u otra con respecto al concepto en reflexión, se puede apreciar que este término aparece con un sentido totalmente peyorativo que es utilizado para denostar las actividades que desde una perspectiva es considerada inútil o poco productiva. Un ejemplo de este sentido, son las palabras de Francis Bacon. Este filósofo inglés señaló en primer lugar que las ciencias de su tiempo vienen de los griegos y se caracteriza por versar sobre la enseñanza y las discusiones o discursos. Luego sostuvo que la sabiduría de los griegos estaba dividida en dos bandos: los que cobraban por enseñar -es evidente que aquí hace alusión a los 
sofistas- y los que no cobraban -es decir, los aletheicos-. Por último, señaló que a pesar de sus diferencias, toda esa sabiduría o ciencia tenía algo en común, era una actividad de ociosos: “... de suerte que se pudo aplicar a todas esas doctrinas, el epigrama bastante justo de Denys referente a Platón: 'todo eso son discursos de viejos ociosos a jóvenes sin experiencia'...” (Bacon 1984, p. 63). Lo que sostiene aquí Bacon es interesante, ya que aseverar que tanto sofistas y los otros, es decir, los filósofos, los aletheicos, los buscadores de la verdad, los sabios, etc., tienen en común practicar una actividad que denomina de ociosos; pero muchos en el mundo griego practicaban esta actividad, entonces surge la interrogante aquí: ¿esto era positivo o negativo? Parece que la intención de Bacon es señalar un rasgo negativo de esta actividad ya que, como se sabe, Bacon va a tomar una actitud demarcacionista contra toda la filosofía anterior y, en especial, la filosofía de la naturaleza, al sostener su teoría de los idola y su método de las tres tablas. Empero, a pesar de su intención donde aparenta denostar, brota en Bacon una posición antitética frente al término en cuestión, pero antitética desde el rasgo y predominio negativo.

Siguiendo con la visión negativa, se puede afirmar que la palabra pereza aparece en Immanuel Kant como una concepción negativa, es decir, cuando está ligada a la concepción de la minoría de edad, donde el hombre no asume responsabilidades como la de pensar por sí mismo: "Pereza y cobardía son las causas merced a las cuales tantos hombres continúan siendo con gusto menores de edad durante toda su vida" (Kant 2013, p. 87). Esta crítica a la pereza vuelve a aparecer en el tercer ejemplo de deber que pone cuando elabora sus cuatro dilemas morales: "Pero se pregunta si su máxima de dejar sin cultivo sus dotes naturales se compadece, no sólo con su tendencia a la pereza, sino también con eso que se llama deber" (Kant 1980, p. 75). Aquí se puede observar que el autor utiliza pereza como antítesis del deber para luego señalar la negatividad de esta acción cuando indica que: "aunque el hombre -como hace el habitante del mar del Surdeje que se enmohezcan sus talentos y entregue su vida a la ociosidad, al regocijo y a la reproducción; en una palabra, al goce" (Kant, p. 75). Al respecto, se puede observar que la pereza u ociosidad son actitudes que pone en cuestión la moralidad del hombre, es decir, contrario al deber que postula el autor. Esta posición negativa del término ocio es reforzada cuando en el libro Crítica de la razón pura, el autor aborda el tema de la verdad y aparece una concepción de ocio ligada a la actividad cognoscitiva, pero esta relación se da dentro de la esfera negativa del término:

Saber lo que racionalmente puede pedirse es por sí solo prueba harto evidente de cordura y de talento; porque si la prueba es absurda en sí y requiere respuestas ociosas, no sólo deshonra al que la hace, sino que a veces produce el inconveniente de precipitar en el absurdo al que sin pensar responde y dar de esta suerte el triste espectáculo de dos personas que, como decían los antiguos, ordeña la una al chivo mientras que la otra pone debajo la criba. (Kant, 1984, p. 135)

Y, para terminar con esta posición negativa, se puede señalar que, aunque no se menciona directamente el término ocio, se hace mención de palabras y frases de similar significado cuando Frederick W. Taylor trabaja las características de la administración científica. Aquí se señala, por ejemplo, que el trabajo lento del obrero es uno de los peores males: 
Trabajar menos de lo posible, es decir, trabajar lentamente con todo propósito de manera que no se llegue a hacer todo el trabajo correspondiente a una jornada (...) es algo casi universal en los establecimientos fabriles, e impera también en gran parte en los oficios de la construcción... (Taylor, 1975, p. 22)

Hasta donde se puede observar, el trabajo lento afecta la producción y, por ende, es mal visto en el sistema administrativo y empresarial, en consecuencia, el autor pretende terminar con este tipo trabajo: "La eliminación del bajo rendimiento y de las diversas causas que deciden el trabajo lento rebajaría tanto el costo de la producción que nuestros mercados, ..., se ampliarían muchísimo... y podríamos competir en condiciones más que iguales con nuestros rivales" (Taylor, 1975, p. 23). Luego, en su afán de buscar la máxima prosperidad, tanto para el patrón como para el obrero, señalará que las causas de este trabajo lento son la mentira de los trabajadores, los defectuosos sistemas de administración y los métodos ineficientes. Esta situación se podrá superar con la administración científica, sin embargo, cuando hace este análisis, el autor señalará que trabajo lento o bajo rendimiento del trabajador es igual a haraganeo: "Este haraganeo o 'bajo rendimiento' tiene su origen en dos causas” (Taylor, p. 26). Calificará a la primera causa como "poco rendimiento natural" (Taylor, p. 26), y en la segunda causa como "bajo rendimiento sistémico" (Taylor, p. 26).

Este haraganeo natural o sistémico que pretende superar este autor lo tratará más adelante con el nombre de pereza: "la pereza natural del hombre es algo serio, pero el mayor mal de que padecen tanto los trabajadores como los patronos es el bajo rendimiento sistémico" (Taylor, 1975, p. 28). Este haraganeo es el que pretende superar con sus principios de la administración científica: "cabe suprimir en gran parte tanto la holgazanería natural como el bajo rendimiento sistémico de trabajo" (Taylor, p. 28).

Reflexionando sobre lo expuesto por Frederick W. Taylor, se puede afirmar que el trabajo o rendimiento lento, que se expresa en una modalidad natural o sistémica, es asociada también a las nociones de haraganeo, pereza u holgazanería, los cuales, a su vez, se les puede ligar al concepto de ocio. Este ocio no mencionado que pretende eliminar el autor con la aplicación de los preceptos de una administración científica. Sin embargo, aquí hay que hacer una acotación, el autor tiene una posición negativa del concepto que se revisa y que en sus dos modalidades afecta la productividad de una empresa, los cuales pretenderá superar con procedimientos científicos.

Sobre la posición negativa del término ocio y tomando en cuenta todas las opiniones autorizadas que se han descrito y que parecen similares unas a otras, a pesar de pertenecer a diferentes épocas y contextos, las cuales son repetidas como si fueran un eco a través de los tiempos cuando las personas comunes y corrientes afirman u opinan: "El ocio es la madre de todos los vicios”, es necesario preguntarse: ¿Es cierto esto? Parece que muchos están de acuerdo, pero en la actividad filosófica existe también una visión diferente y totalmente opuesta al sentido común y negativo del término ocio y de los significados semejantes. 


\section{CONCEPCIÓN POSITIVA DEL TÉRMINO OCIO}

En el quehacer filosófico hay una percepción muy original acerca de la utilidad del ocio o solaz. Esta posición se va a analizar aquí a través de las afirmaciones de Platón, Aristóteles y Cicerón.

Cuando Platón analizó en Critias la situación en la cual vivían los antiguos pobladores de la mítica Atlántida afirmó que la producción de medios de subsistencia de una comunidad trae como consecuencia el descuido de otras actividades: "Dada la escasez de subsistencias para el sostenimiento de la vida... para nada se cuidaron de los sucesos que en otro tiempo se habían realizado" (Platón 1965, p. 957). Luego de esta observación, el filósofo de los hombros anchos sostendrá que el ocio es la madre de muchas cosas positivas para la sociedad y cultura humana:

El estudio y la historia de las cosas antiguas se introdujeron con el ocio en las ciudades, cuando cierto número de ciudadanos, teniendo aseguradas las cosas necesarias para la vida, no tuvieron después que preocuparse desde este punto de vista. (Platón 1965, p. 957)

Por consiguiente, se puede resumir aquí que para Platón, por lo menos en el texto mencionado, el ocio citadino no tiene un sentido negativo sino un valor positivo. Se analiza aquí que este ocio nace después del trabajo, es decir, después de que el hombre resuelve la producción de sus medios de subsistencia.

De la misma opinión de Platón es, también, Aristóteles cuando sostuvo que el ocio es la partera de las ciencias, ojo, no dijo que la filosofía es la madre de las ciencias, sino el ocio o solaz:

De ahí que, una vez constituidas todas las técnicas, se descubrieron las ciencias que no tienen por objeto ni el placer ni la necesidad. Se originaron, en primer lugar, en los países donde los hombres gozaban de ocio. Por esta razón las matemáticas nacieron en Egipto, porque en ese país le fue concedido el ocio a la clase sacerdotal. (Aristóteles, 1986, p. 93)

Estas mismas afirmaciones sobre el ocio son traducidas al castellano de forma muy similar en una edición trilingüe, las cuales no cambiaron el sentido de la traducción anterior:

De aquí que, constituidas todas estas artes, fueron descubiertas las ciencias que no se ordenan al placer ni a lo necesario; y lo fueron primero donde primero tuvieron que vagar los hombres. Por eso las artes matemáticas nacieron en Egipto, pues allí disfrutaba de ocio la clase sacerdotal. (Aristóteles 1987, p. 9)

En consecuencia, se interpreta que Aristóteles, al igual que Platón, piensa que el ocio o solaz aparece cuando la sociedad o el Estado han resuelto sus problemas de producción de los medios de subsistencia. Florece, además, en el sector dirigente de la sociedad y que es la que está más preparada para aprovechar el tiempo libre en otras actividades.

En otra versión sobre el tema, se puede apreciar la opinión sobre el ocio de Cicerón (2003) cuando al disertar acerca de la naturaleza de los dioses llega a afirmar que "todas 
las enseñanzas filosóficas tienen una aplicación en la vida" (p. 64) y, luego, alcanza a sostener la importancia del solaz para el filosofar:

Por lo demás, si alguien se pregunta qué motivo nos impulsó a poner por escrito estas cosas tan tardíamente, no hay nada que podamos revelar con tanta facilidad. Resulta que, como languidecíamos a consecuencia de la ociosidad y era tal la situación del Estado que su gobierno había de estar, necesariamente, bajo el designio y cuidado de una sola persona, di en pensar, por primera vez, que a nuestros hombres se les habría de explicar filosofía, en beneficio del propio Estado, estimando que era de gran interés para la honra y alabanza de nuestra ciudad que unos asuntos tan serios e ilustres tuvieran su lugar también en la literatura latina. (Cicerón 2003, p. 64)

Se puede observar en Cicerón una concepción antitética de ocio, donde se manifiestan los rasgos negativos y positivos, pero con una preeminencia de estos últimos al asociar la actividad filosófica a la ociosidad en provecho del Estado.

En resumen, sobre el enfoque positivo del concepto ocio, se puede apreciar que Platón, Aristóteles y Cicerón tenían una gran estima hacia el ocio porque es el punto de partida de diferentes tipos de quehaceres en el hombre, desde los más simples hasta los más sublimes. Tal es el caso que, desde la perspectiva de estos filósofos, se puede apreciar que el ocio es una actividad productiva y partera de las artes o técnicas, de las ciencias y la filosofía, del gobierno y la política. Señalan, en pocas palabras, que el ocio es productivo; pero, además, es necesario satisfacer primero las necesidades básicas dentro de un orden social determinado para poder dedicarse después a este gran solaz, es decir, al tiempo libre para disertar libremente, para filosofar, el cual es uno de los productos del ocio.

\section{CONCLUSIÓN, REFLEXIÓN Y POSIBILIDAD ANTITÉTICA DEL TÉRMINO OCIO}

A manera de conclusión y después de las observaciones realizadas, se puede aseverar que existe una visión antitética del ocio o solaz, tanto en la filosofía como en las ciencias, la poesía y las artes. Este enfoque tiene implicancias en la concepción de la sociedad y el Estado. El argumento que sostiene la tesis antitética planteada pasa por dos apreciaciones del concepto que son totalmente opuestas y son sostenidas, desde un frente, por Hesíodo, Séneca, Michael de Montaigne, Francis Bacon, Immanuel Kant y Frederick W. Taylor donde se confunde o combina con la opinión común y corriente que valoran negativamente el ocio o la actitud ociosa; mientras que, desde el otro frente, Platón, Aristóteles y Cicerón representan una apreciación positiva del término, la cual, por lo general, es inesperada por la opinión común. Según esta última estimativa, se puede dilucidar que el ocio es un componente necesario para la realización de actividades o trabajos distintos a los manuales, pero debe surgir después que la comunidad o sociedad humana haya resuelto sus problemas de producción que satisfagan las necesidades fundamentales de existencia y gobierno de un Estado o, por lo menos, del grupo o comunidad, casta o clase social implicados directamente en hacer uso de este término tan vituperado. En casos como estos han surgido propuestas teóricas y sociales que formulan una sociedad basada en principios donde el asalariado tenga una vida más placentera con el mayor tiempo libre que se pueda. Esto trae como consecuencia que, en los últimos 
tiempos, aparezca en el escenario actual, tanto en las reivindicaciones sociales, políticas y económicas, la necesidad de tener más tiempo libre, el imperativo de reducir las horas de trabajo, la comodidad de vivir en una sociedad basada en el principio de la felicidad y el amor, y rechazar el principio de rendimiento que agobia la vida de la mayoría de los seres humanos, aunque exista el deber de producir para crear una sociedad que satisfaga las necesidades de todos.

El significado de los términos varía con los cambios de época, son equívocos, y el término ocio no es ajeno a esta determinación. Por ejemplo, en la actual sociedad "globalizada" ya se vislumbraba que aquella persona que no sale de su casa o que permanezca en ella, por un sinfín de motivos, no tiene que ser calificada necesariamente de ociosa o proclive a ella porque todos saben que existen muchas personas que son muy productivas en casa no solamente por las tareas del hogar, sino porque se generan ingresos necesarios para la manutención de la familia, incluso se han hecho grandes fortunas desde el trabajo en casa o en un garaje. En ella, personas en apariencia improductiva han resultado ser personas de mucho beneficio para la sociedad y humanidad. Otro ejemplo más se visualiza cuando, en el contexto actual de plena pandemia producida por el Covid19, los gobiernos recomiendan a las personas o ciudadanos responsables quedarse en casa, la respuesta es diversa, gran parte de la población obedece, pero muchas otras no siguen las recomendaciones con aceptables o inaceptables justificaciones. Los calificativos que se les pueden dar a unos y a otros son diversos, pero lo anecdótico del escenario es que aparece el término ocio. Por ejemplo, hay personas que antes no hacían nada: no trabajaban, no estudiaban, no salían a ayudar en las compras, no ayudaban en las tareas del hogar y, en consecuencia, eran fácilmente calificadas de vagos u ociosos; mientras, las demás eran calificadas de buenas personas o ciudadanos. Sin embargo, en las actuales circunstancias, esto ya no se cumple porque ahora una persona que sale a ganarse el pan con el sudor de su frente, espalda y brazos, es decir, su cuerpo, puede ser mal vista y calificada negativamente por ser el eslabón de una cadena de contagio que puede poner en peligro a toda la comunidad -;Oh, ironía!-; por otro lado, aquella persona que no hacía nada en su casa y, por su vida, sigue en la misma situación y no sale de su casa, cumpliendo con las normativas del Estado de confinamiento y distanciamiento social, puede ser ahora visto como buen ciudadano y calificado como persona responsable.

Queda entonces demostrada la posibilidad antitética del término ocio en la evolución de la palabra a través de la historia humana, con las tesis y argumentos de ilustres pensadores, y en el contexto actual, a través de una pandemia de proporciones inimaginables, en el análisis de especialistas y el imaginario colectivo de gente común o sencilla. El término ocio tiene un significado antitético, es decir, positivo y negativo, para calificar las conductas de las personas, tanto individual como colectivamente, uno no excluye al otro y tampoco es superior, incluso en la valoración moral o social.

\section{REFERENCIAS BIBLIOGRÁFICAS}

Aristóteles. (1986). Metafísica (2a ed.). Buenos Aires, Argentina: Editorial Sudamericana.

Aristóteles (1987). Metafísica de Aristóteles: texto trilingü̈e (Vol. 1., $2^{\mathrm{a}}$ ed., $1^{\mathrm{a}}$ reimpr.). Madrid, España: Editorial Gredos. 
Bacon, F. (1984). Novum organum. Madrid, España: Sarpe.

Cicerón. (2003), Sobre la naturaleza de los dioses. Barcelona, España: Editorial Gredos.

Diccionario latino-español español-latino. (1958). Barcelona, España: Editorial Dalmau y Jover S.A.

Engels, F. (1971). Del socialismo utópico al socialismo científico. Moscú, Rusia: Editorial Progreso.

Hesíodo. (2000). Obras y fragmentos. Madrid, España: Editorial Gredos.

Kant, M. (1980). Fundamentación de la metafísica de las costumbres (6a ed.). Madrid, España: Espasa-Calpe.

Kant, I. (1984), Crítica de la razón pura (2 Vols.). Buenos Aires, Argentina: Ediciones Orbis.

Kant, I. (2013). Contestación a la pregunta: ¿Qué es la Ilustración? En R. Aramayo (Ed.), ¿Qué es la Ilustración? Y otros escritos de ética, política y filosofía de la bistoria ( $2^{\mathrm{a}} \mathrm{ed} ., \mathrm{pp} .85-$ 98), Madrid, España: Alianza Editorial.

Montaigne, M. (1898), Ensayo (Vol. 1). Recuperado de: ebookbrowse.com/montaignemichel-de-ensayos-libro-1doc-d424471297.

Platón. (1965). Diálogos: La República o el Estado. Madrid, España: EDAF.

Séneca. (2005). Tratado de la brevedad de la vida (2 ${ }^{\mathrm{a}}$ ed.). Madrid, España: Mestas Ediciones.

Taylor, F. W. (1975). Principios de la administración científica (18 a ed.) y En (Eds.). Principios de la administración cientifica - Administración industrial y general (pp. 1-124), México: Herrero Hermanos Sucs. S. A.

CITAR COMO:

Bretoneche Gutiérrez, L. A. (2020). Enfoque antitético del ocio. Puriq, 2(3), 176-185. https://doi.org/10.37073/puriq.2.3.85 